PROCEEDINGS OF THE

AMERICAN MATHEMATICAL SOCIETY

Volume 129, Number 10, Pages 2913-2917

S 0002-9939(01)06182-2

Article electronically published on April 24, 2001

\title{
A FAMILY OF KÄHLER-EINSTEIN MANIFOLDS AND METRIC RIGIDITY OF GRAUERT TUBES
}

\author{
RÓBERT SZŐKE
}

(Communicated by Mohan Ramachandran)

\begin{abstract}
In this paper we explain how the so-called adapted complex structures can be used to associate to each compact real-analytic Riemannian manifold a family of complete Kähler-Einstein metrics and show that already one element of this family uniquely determines the original manifold. The underlying manifolds of these metrics are open disc bundles in the tangent bundle of the original Riemannian manifold.
\end{abstract}

Recall first the notion of adapted complex structures (cf. [7, [12, 16]). Let $(M, g)$ be a complete real-analytic Riemannian manifold. If $\gamma$ is a geodesic in $M$, we can define a map $\psi_{\gamma}: \mathbb{C} \rightarrow T M$ by

$$
\psi_{\gamma}: \sigma+i \tau \mapsto \tau \dot{\gamma}(\sigma)
$$

If $r \in(0, \infty]$, we let $T^{r} M$ denote the open disc bundle in $T M$ consisting of tangent vectors of norm less than $r$ (note that we allow $r$ to be infinite). A complex structure on $T^{r} M$ is said to be adapted with respect to $g$ if $\psi_{\gamma}$ is holomorphic on $\psi_{\gamma}^{-1}\left(T^{r} M\right)$ for each geodesic $\gamma$. We shall usually omit the phrase "with respect to" if it is obvious which metric is being discussed. The manifolds $T^{r} M$ are also called Grauert tubes since Grauert used such manifolds in his famous result to show that each real-analytic manifold admits a real-analytic embedding to a euclidean space.

Adapted complex structures were discovered by studying certain global solutions of the complex homogeneous Monge-Ampère equation on Stein manifolds (cf. 2], [7], 12], [14]). Their basic properties were treated in [7, 12], 16]. Among others, one has an existence result: if $(M, g)$ is a compact real-analytic Riemannian manifold, then there exists an $r \in(0, \infty]$ such that $T^{r} M$ carries an adapted complex structure [7], [16]. Also the adapted complex structure is uniquely determined by $(M, g)($ 7], 12]).

From now on we shall take $(M, g)$ to be a compact Riemannian manifold and use $R$ to denote the largest element of $(0, \infty]$ such that $T^{R} M$ supports an adapted complex structure.

It was shown in [12] that the energy function $E$ on $T M$ which assigns to each tangent vector the half of its norm-square with respect to the metric $g$ is strictly plurisubharmonic on $T^{R} M$. Thus another theorem of Grauert implies that $T^{R} M$ is a Stein manifold. Hence $T^{r} M$ is relatively compact and strictly pseudoconvex whenever $0<r<R$.

Received by the editors February 2, 2000.

2000 Mathematics Subject Classification. Primary 32Q15, 53C35.

Key words and phrases. Kähler-Einstein metrics, adapted complex structures, Grauert tubes. 
Now it follows from the work of Cheng and Yau [5, 13] that a strictly pseudoconvex relatively compact domain $\Omega$ in a Stein manifold $X$ admits a complete Kähler-Einstein metric. The biholomorphisms of $\Omega$ will be isometries, and moreover, this metric is unique if we normalize its Einstein constant to be -1 .

This gives us the following result.

Theorem 1. Let $(M, g)$ be a compact real-analytic Riemannian manifold. Then $T^{r} M$ carries a complete Kähler-Einstein metric $h_{r}$ with Einstein constant -1 whenever $0<r<R$.

Remarks. (1) Since the adapted complex structure on $T^{r} M$ is unique, the natural map of $T^{r} M$ to itself induced by an isometry of $(M, g)$ is a biholomorphism of $T^{r} M$ and hence an isometry of the Kähler-Einstein metric $h_{r}$. It follows that the isometry group of $(M, g)$ injects into the isometry groups of $\left(T^{r} M, h_{r}\right)$ and $\left(M,\left.h_{r}\right|_{M}\right)$. We deduce that if $(M, g)$ is an isotropy irreducible homogeneous space, then $g$ and $\left.h_{r}\right|_{M}$ are homothetic. In general, however, there seems to be no reason to believe that this holds.

(2) It was shown in [16] that if $(M, g)$ is a compact symmetric space $U / K$, then an adapted complex structure exists on the entire tangent bundle. If the rank of the symmetric space is 1 , the generic orbit of the $U$ action on $T M$ has codimension one, so in this case it follows that for each $r \in(0, \infty)$ we have a complete KählerEinstein metric of cohomogeneity one on $T^{r} M$. When $M$ is the two-sphere, this metric is shown to exist in the course of a classification of Kähler-Einstein metrics of Bianchi IX type [6].

Now let $M$ be the unit circle $S^{1}$. As one easily sees, for any $0<r<\infty$ the tube $T^{r} S^{1}$ with its adapted complex structure can be identified with the annulus $A_{r}$ whose center is the origin and has radii $e^{-r}$ and $e^{r}$. As is well known, the moduli space of the annuli on the complex plane is parametrized by one real parameter. Now the annuli $A_{r}$ as $r \in(0, \infty)$ chooses precisely one representative from each equivalence class, and one could consider them a canonical form.

Now the annuli $A_{r}$ are not only complex manifolds but they possess a symplectic structure (the standard area form) and also a metric structure (a unique complete hyperbolic metric of constant -1 curvature).

As one easily sees, the biholomorphic, symplectic and metric classification of the annuli $A_{r}$ are all the same. The tubes $T^{r} M$ show remarkable similar rigid behaviour. The study of their biholomorphic rigidity was initiated in [17] and continued by D. Burns 3, in a series of papers by S. J. Kan and D. Ma [9, 10, 11] and finally D. Burns and R. Hind [4] completed the biholomorphic classification.

Theorem 2 ([3], 4]). Let $(M, g)$ and $(N, h)$ be compact, real-analytic Riemannian manifolds with adapted complex structures on $T^{R} M$ and $T^{S} N$. Let $0<r<R$, $0<s<S$. Suppose $\Phi$ is a biholomorphism between the manifolds $T^{r} M$ and $T^{s} N$. Then there exists an isometry

$$
\varphi:(M, s g) \rightarrow(N, r h)
$$

such that $\Phi=\varphi_{*}$.

The symplectic classification of Grauert tubes is far from being well understood. A result of J. C. Sikorav [15] shows that tubes over flat tori are symplectomorphic iff they are biholomorphic. 
Our concern in this paper is the metric rigidity of the tubes $\left(T^{r} M, h_{r}\right)$. Unfortunately, the Cheng-Yau metric is highly nonexplicit and is not easy to work with. First we start with a preliminary lemma.

Lemma 3. Let $(M, g)$ be a compact connected real-analytic Riemannian manifold with universal cover $(\tilde{M}, \tilde{g})$. Then, whenever $0<r<R$, the complex manifold $T^{r} \tilde{M}$ is not biholomorphic to a product of complex manifolds.

Proof. A result of Huckleberry [8] shows that a connected strictly pseudoconvex relatively compact domain $D$ in a complex manifold cannot be the total space of a locally trivial holomorphic fibration, so in particular cannot be biholomorphic to a product of complex manifolds.

The only use made of relative compactness in his proof is as follows. Suppose there is a given sequence of holomorphic maps $\psi_{j}$ from a polydisc $\Delta$ to $D$, such that $\psi_{j}(0)$ converges to a point $p_{0} \in \partial D$. If $D$ is relatively compact, then Montel's theorem implies the convergence of a subsequence of $\psi_{j}$ to a holomorphic map $\psi: \Delta \rightarrow \bar{D}$, where $\psi(0)=p_{0}$. Now strict pseudoconvexity at $p_{0}$ and the maximum principle shows that $\psi$ is identically equal to $p_{0}$. It follows that for each $z \in \Delta$, a subsequence of $\psi_{j}(z)$ converges to $p_{0}$. It is this result that we must establish.

In our situation $D=T^{r} \tilde{M}$ will be connected and strictly pseudoconvex, but not relatively compact in $T^{R} \tilde{M}$ unless $M$ has finite fundamental group. However, as explained earlier, we have a holomorphic covering $\pi: T^{R} \tilde{M} \rightarrow T^{R} M$; moreover, this restricts to a covering $D \rightarrow H=T^{r} M$ mapping $\partial D$ onto $\partial H$. Now $H$ is relatively compact in $T^{R} M$ so the argument of the preceding paragraph shows that a subsequence $\pi \psi_{j_{k}}$ of $\psi_{j}$ converges to the constant map from $\Delta$ to $\partial H$ with value $\pi p_{0}$. Let $V$ be a neighborhood of $\pi p_{0}$ in $T^{R} M$ such that $\pi^{-1}(V)$ is a disjoint union of open sets $V_{\alpha}$, each containing precisely one element of $\pi^{-1}\left(\pi p_{0}\right)$. Let $V_{0}$ be the open set in this family containing $p_{0}$. As $\psi_{j}(\Delta)$ is connected and $\psi_{j}(0)$ converges to $p_{0}$ by hypothesis, we see that for sufficiently large $k$ the subsequence $\psi_{j_{k}}$ has image contained in $V_{0}$. We can make $V_{0}$ arbitrarily small by shrinking $V$ suitable, so we deduce that for each $z \in \Delta$ the subsequence $\psi_{j_{k}}(z)$ converges to $p_{0}$.

The remainder of the proof proceeds as in 8 .

Theorem 4. Let $(M, g),(N, h)$ be compact, connected real-analytic Riemannian manifolds such that $T^{R} M$ and $T^{S} N$ support an adapted complex structure. Let $0<r<R, 0<s<S$ and $\Phi$ be a diffeomorphism between the manifolds $T^{r} M$ and $T^{s} N$. Then the following statements are equivalent:

1) $\Phi:\left(T^{r} M, h_{r}\right) \rightarrow\left(T^{s} N, h_{s}\right)$ is an isometry,

2) $\Phi$ is biholomorphic or antibiholomorphic,

3) There exists a $\varphi:(M, s g) \rightarrow(N$, rh $)$ isometry such that $\Phi \equiv \varphi_{*}$ or $\Phi \equiv-\varphi_{*}$.

Proof. For any manifold $L$ denote by $\sigma$ the self map of $T L$ that multiplies each vector with negative one. As shown in 12, this map is antiholomorphic with respect to the adapted complex structure. Also, the uniqueness of the adapted complex structure implies that the complex structure adapted to $g$ will also be adapted to any metric that is homothetic to $g$. These together with Theorem 1 and Remark (1) give the equivalence of 2) and 3).

Now suppose $\Phi$ is antibiholomorphic. Then $\sigma \circ \Phi$ is a biholomorphism and the uniqueness of the Cheng-Yau metric implies that this map is also an isometry. It was observed by D. Burns [3] that $\sigma$ is also an isometry of the Cheng-Yau metric $h_{s}$. This yields that 2) implies 1 ). 
Now assume that $\Phi$ is an isometry. The metrics $h_{r}$ and $h_{s}$ lift to Kähler metrics on $T^{r} \tilde{M}$ and $T^{s} \tilde{N}$. If $h_{r}$ or $h_{s}$ is locally reducible, then the associated Kähler metric on $T^{r} \tilde{M}$ or $T^{s} \tilde{N}$ is reducible, contradicting Lemma 3 . We see that $h_{r}$ and $h_{s}$ are Kähler metrics with nonvanishing Ricci tensor (by definition) and are not even locally reducible.

If $h_{r}$ (and thus $h_{s}$ ) is locally symmetric, then $T^{r} \tilde{M}$ and $T^{s} \tilde{N}$ are irreducible symmetric spaces of negative Ricci curvature, i.e., they are symmetric spaces of Helgason's noncompact type. The isometry $\Phi$ lifts to an isometry between these globally symmetric spaces and so it must be biholomorphic or antibiholomorphic giving 2).

If $h_{r}$ is not locally symmetric, then the preceding paragraph shows that for each metric the only covariant constant two-forms are multiples of the Kähler form. Berger's classification of holonomy groups shows that the existence of other covariant constant two-forms reduces the holonomy to the symplectic group and this forces the Ricci tensor to vanish [1].

Note also that the volume forms of $h_{r}$ and $h_{s}$ are powers of the Kähler forms (up to multiplication by a constant depending only on the dimension of $M$ ). We deduce that $\Phi$ will preserve the Kähler forms up to a sign, so there is either a holomorphic or antiholomorphic bijection between $T^{r} M$ and $T^{s} N$.

As a corollary we get the following.

Corollary 6. Let $(M, g)$ be a compact real-analytic Riemannian manifold. Suppose that for $R \in(0, \infty], T^{R} M$ admits an adapted complex structure. If $S, Q$ are distinct positive numbers less than $R$, then the Kähler-Einstein manifolds $\left(T^{S} M, h_{S}\right)$ and $\left(T^{Q} M, h_{Q}\right)$ are not isometric.

\section{ACKNOWLEDGEMENTS}

The author thanks A. S. Dancer and D. Burns for very stimulating discussions and the Mathematical Institute of Oxford University for its hospitality. This research was partially supported by the Max Planck Institut für Mathematik Bonn, the Hungarian Research grants FKFP 0230/1999, OTKA T032478 and the Eötvös fellowship.

\section{REFERENCES}

1. A. Besse, Einstein manifolds, Ergebnisse der Math. und ihrer Grenzgebiete, Springer-Verlag, 1987. MR 88f:53087

2. D. Burns, Curvatures of Monge-Ampère foliations and parabolic manifolds, Ann. Math. 115 (1982), 349-373. MR 84a:32031

3. - On the uniqueness and characterization of Grauert tubes, in: Complex Analysis and Geometry, eds: V. Ancona, A. Silva, 1995, pp. 119-133. MR 97e:32016

4. D. Burns and R. Hind, Symplectic geometry and the uniqueness of Grauert tubes, Preprint, xxx.lanl.gov, math.CV/0010299

5. S.Y. Cheng and S. T. Yau, On the existence of a complete Kähler metric on non-compact complex manifolds and the regularity of Fefferman's equation, Comm. Pure Appl. Math. 33 (1980), 507-544. MR 82f:53074

6. A. S. Dancer and I. A. B. Strachan, Kähler-Einstein metrics with SU(2) action, Math. Proc. Camb. Phil. Soc. 115 (1994), 513-525. MR 95g:53053

7. V. Guillemin and M. Stenzel, Grauert tubes and the homogeneous Monge-Ampère equation I, J. Diff. Geom. 34 (1991), 561-570. MR 93e:32018

8. A. Huckleberry, Holomorphic fibrations of bounded domains, Math. Ann. 227 (1977), 61-66. MR 55:8417 
9. S. J. Kan, On the rigidity of non-positively curved Grauert tubes, Math. Z. 229 (1998), no. 2, 349-363. MR 99i:32011

10. S. J. Kan and D. Ma, On rigidity of Grauert tubes over Riemannian manifolds of constant curvature, Preprint 1997.

11. - On rigidity of Grauert tubes over locally symmetric spaces, J. Reine Angew. Math. 524 (2000), 205-225. CMP 2000:15

12. L. Lempert and R. Szőke, Global solutions of the homogeneous complex Monge-Ampère equation and complex structures on the tangent bundle of Riemannian manifolds, Math. Ann. 291 (1991), 689-712. MR 92m:32022

13. N. Mok and S. T. Yau, Completeness of the Kähler-Einstein metric on bounded domains and the characterisation of domains of holomorphy by curvature conditions, in "The mathematical heritage of Henry Poincaré", Proc. Symp. Pure Math. 39 (1983), 41-59. MR 85j:53068

14. G. Patrizio and P. M. Wong, Stein manifolds with compact symmetric center, Math. Ann. 289 (1991), 355-382. MR 92e:32009

15. J. C. Sikorav, Rigidité symplectique dans le cotangent de $T^{n}$, Duke 59 (1989), 759-763. MR 91e:58063

16. R. Szőke, Complex structures on the tangent bundle of Riemannian manifolds, Math. Ann. 291 (1991), 409-428. MR 93c:53023

17. R. Szőke, Automorphisms of certain Stein manifolds, Math. Zeitschr. 219 (3) (1995), 357-387. MR 96e:32028

Department of Analysis, Eötvös University, Kecskeméti u. 10-12, 1053 Budapest, HUNGARY

E-mail address: rszoke@cs.elte.hu 